\title{
Acupuncture and Chinese Medicine in the Modern Age: WFAS Sixth World Conference on Acupuncture
}

\author{
Gold Coast Convention and Exhibition Centre in Australia, \\ October 29-31, 2004
}

\author{
Sheng-Xing Ma \\ Department of Obstetrics and Gynecology, David Geffen School of Medicine at University of California Los \\ Angeles, Harbor-UCLA Medical Center, USA
}

\section{General Organization}

The Sixth World Federation of Acupuncture Moxibustion Societies (WFAS) Academic Conference on Acupuncture was organized at the Gold Coast Convention and Exhibition Centre in Australia, October 29-31, 2004. WFAS is the official nongovernmental organization advising the World Health Organization (WHO) on acupuncture, and has successfully held five World Academic Conferences on Acupuncture and 11 International Acupuncture Symposia. The Sixth World Conference on Acupuncture was held by the Australian Acupuncture and Chinese Medicine Association Ltd, cosponsored by WFAS and WHO.

WFAS 2004 attracted a wide range of academics and practitioners in acupuncture globally from 28 countries and areas. There were 251 abstracts presented in the conference including 147 oral presentations and many posters.

\section{Conference Missions}

The conference focused primarily on acupuncture including body acupuncture, ear acupuncture and diagnostic techniques, with an emphasis on traditional Chinese medicine (TCM) theory and concepts as well as the modern scientific approach. WFAS 2004 provided a timely focus on several themes which our profession must focus on and address. The program covered various subjects in the field of clinical practice of acupuncture, policy, regulation, research, education, standards, safety and much more.

For reprints and all correspondence: Department of Obstetrics and Gynecology, David Geffen School of Medicine at University of California Los Angeles, Harbor-UCLA Medical Center, Los Angeles, CA, USA. E-mail:ma@humc.edu
The audience received updated information on recent advances in scientific and clinical research on acupuncture. Almost all medical practitioners had previous experience in acupuncture and actively took part in the discussion of scientific reports. Experimental results have provided the bridge between acupuncture mechanisms and their clinical, therapeutic effects. The presentations and discussions advanced the knowledge of acupuncture practices, education and standards, and enhanced communication between research scientists and clinicians, as well as administrators and acupuncture practitioners.

It is worth noting that several reports were presented on statistical methods for evaluating study results of acupuncture/ TCM trials: e.g. Hierarchical Linear Model (HLM) and Acupuncture Research, by $\mathrm{Ke} \mathrm{Li}$; also Acupuncture for Arthritis-Meta-analysis, presented by Professor Zhigao Jin, as examples of analysis of smaller sample sizes in acupuncture research. These studies are representative of a new trend that will strengthen our profession, namely, the participation in TCM research of specialists from other fields in medicallyrelated research, from clinicians to western research scientists, including, in this instance, mathematical statisticians. Such studies also incorporate computer-based modeling.

\section{International Practices, Education and Research on Acupuncture}

In China and other East Asian countries, acupuncture and TCM have historical and cultural integration as fundamental health and medical modes. There is general public acceptance and also governmental support for TCM. Professor Xuetai Wang, Honorary President of WFAS, gave the opening keynote address: Discussion of the Distinguishing Features of Acupuncture and Moxibustion. He considered the unique

(C) The Author (2005). Published by Oxford University Press. All rights reserved.

The online version of this article has been published under an open access model. Users are entitled to use, reproduce, disseminate, or display the open access version of this article for non-commercial purposes provided that: the original authorship is properly and fully attributed; the Journal and Oxford University Press are attributed as the original place of publication with the correct citation details given; if an article is subsequently reproduced or disseminated not in its entirety but only in part or as a derivative work this must be clearly indicated. For commercial re-use, please contact journals.permissions@oupjournals.org 
features of acupuncture and moxibustion as therapeutic modes under three general themes: their display, their significance, and their continuing viability under the ongoing process of modernization. Display is physiologic, giving many diagnostic indicators, and treatment uses acupoints as the loci of treatment under the median-collateral theory of TCM. The thinking mode of TCM is especially significant because the theory allows diagnosis and treatment of all diseases by means of prescribing acupoints and performing manipulation techniques in line with syndrome differentiation. Professor Wang concluded with a discussion of preserving and promoting the distinguishing features of acupuncture and moxibustion in modernization, particularly by the study of meridian-collateral theory and clinical research on the specific actions of acupuncture points.

Professor Zhixiang Shen, Secretary-General of WFAS and Director of the Department of International Cooperation, China State Administration of TCM, addressed the conference on the important process of achieving a system of uniform standards in his lecture Development and Strategic Plan of Acupuncture Standardization Studies. He reviewed work from the last 20 years through the joint efforts of WHO, WFAS and international experts leading to achievements in standardizations of acupuncture nomenclature, clinical studies, training and safety, and to the Encyclopedia of Acupoints. He stressed that the starting point and basic objective of acupuncture standardizations are to optimize the sequence degrees of the acupuncture system to arrive at the best sequence that will produce the best result, not only for the development of acupuncture itself, but also for its internationalization.

James Flowers, President of the Australian Acupuncture and Chinese Medicine Association, spoke on the need for practitioners of Chinese medicine to organize into a strong professional organization at this time, as we enter a globally- regulated environment. Dr Flowers emphasized that such an organization allows us to define modern professional standards and promote a strong core of ethics with an emphasis on providing health benefits as a public duty. He remarked that we command a body of knowledge which is practical, with intellectual discipline, and aims to benefit the public. In forming a strong professional association we will continue to have a strong role in determining the future of our profession.

There are important differences in public and governmental perceptions about the practice of traditional medicine in developing versus developed countries. Acupuncture and TCM are well-integrated through culture and long history into virtually all countries in South-East Asia. However, in many areas of the developed world, such as Western Europe including the UK and the USA, the predominant medical modality is centered on western medicine. There, TCM and acupuncture are labeled as 'alternative', 'complementary' or 'non-conventional' medicine, and sometimes meet with formal resistance in their practice, such as from conservative western medical colleagues, or by limited governmental recognition.

Dr Seung Hoon Choi, Regional Advisor in Traditional Medicine, WHO/Western Pacific Regional Office (WPRO), described the current first global WHO Traditional Medicine Strategy (2002-2005), which addresses four major challenges to the practice of TM/CAM as defined by WPRO/WHO in Regional Strategy for Traditional Medicine in the Western Pacific (2001-2010). Firstly, the strategy advocates development of a policy to integrate TM/CAM into national health care systems. Second, it states the need for clear scientific evidence for therapeutic efficacy, and the development of regulated quality controls and standards. Third, it desires to protect indigenous TM knowledge, and to increase access and availability of TM/CAM, particularly to poor populations. Fourth, there is the need for therapeutically sound use of appropriate TM/CAM by providers and consumers.

The use of TCM continues to expand rapidly in developed countries, although government funding support varies widely. Dr Alan Bensoussan, Director of the Centre for Complementary Medicine Research at the University of Western Sydney, contrasted lack of Australian government funding for CAM research with that available in the UK and the USA. He described how the University of Western Sydney responded over the last decade by firstly initiating cooperative clinical trials with a wide range of public hospitals, leading to the current formal collaboration with the South Western Sydney Area Health Service (NSW Department of Health) for systematic clinical research in TCM. Clinical trial node areas include cardiovascular disease, aged care, gastroenterology and women's health. There is the long-term goal of developing clinical guidelines for the integration of TCM within modern medical care. However, Dr Bensoussan also commented on sources of institutional resistance to further TCM study, including competition for space and resources in hospital settings, the nervousness of ethics committees and the bias of conservative western medical colleagues.

In order to reach our professional long-term strategic goals, defined in the position papers supported by the WHO, it is necessary to continue both basic science and clinical-based research in TCM and all areas of acupuncture practice. All areas of a research approach benefit by the active participation of patients and volunteer subjects and of doctors and professional practitioners of acupuncture and TCM. Many practitioners at WFAS 2004 from China, Japan, Korea and other East Asian countries presented results of studies done with funding from university and governmental support, and large patient sample sizes were made possible. Large data sets are very useful in defining acupuncture as an evidence-based practice, which is vital if we are to achieve widespread parallel use and incorporation of TCM into Western medical practices, especially in developed countries. Evidence-based practice is also the only way to satisfy the requirements of a globally regulated environment.

\section{Keynote Speakers}

Concurrent sessions in the morning and afternoon always began with a keynote speaker, as detailed below.

\section{Discussion of the distinguishing features of acupuncture and moxibustion}

Date: Friday, October 29, 2004 (Opening ceremony) 
Presenter: Prof. Xuetai Wang, Honorary President of WFAS, China

\section{Nitric Oxide Mediates Acupuncture-Induced Cardiovascular Effects in the Dorsal Medulla-Thalamic Tract}

Date: Friday, October 29, 2004 (Concurrent Presentations) Presenter: Dr Shengxing Ma, Associate Professor, Department of Obstetrics/Gynecology, Harbor-UCLA Medical Center, David Geffen School of Medicine, UCLA, USA.

Future Directions in Acupuncture Research

Date: Friday, October 29, 2004 (Concurrent Presentations) Presenter: Dr Richard Hammerschlag, Research Director, Oregon College of Oriental Medicine, USA.

The History, Culture and TCM Acupuncture for Menopause-The Challenge for Research and a Proposed Solution

Date: Friday, October 29, 2004 (Concurrent Presentations) Presenter: Dr Volker Scheid, Academic Director \& Senior Lecturer, European Institute of Oriental Medicine, UK.

\section{The Therapy of Navel Acupuncture Therapy}

Date: Saturday, October 30, 2004 (Concurrent Presentations) Presenter: Dr Qi Yong, Director of Wenzhou 2nd People's Hospital, Zhejiang, China.

\section{Hierarchical Linear Model (HLM) and Acupuncture Research}

Date: Saturday, October 30, 2004 (Concurrent Presentations) Presenter: Ke Li, Vice-President, Australian Acupuncture and Chinese Medicine Association Ltd, Australia.

\section{The Important Theory of Acupuncture-Application of Muscle Theory}

Date: Saturday, October 30, 2004 (Concurrent Presentations) Presenter: Prof. Xue Ligong, Professor and Senior Consultant, Acupuncture Institute of China Academy of TCM, China.

\section{Into the Fray: Driving TCM Research in Public Hospitals in Australia}

Date: Saturday, October 30, 2004 (Concurrent Presentations) Presenter: Prof. Alan Bensoussan, Head of Chinese Medicine Unit, University of Western Sydney; Director of the UWS Center for Complementary Medicine Research, Australia.

\section{Development and Strategic Plan of Acupuncture Standardization Studies}

Date: Saturday, October 30, 2004 (Concurrent Presentations) Presenter: Prof. Shen Zhixiang, Secretary-General of WFAS \& Director of Department of International Cooperation, China SATCM, China.

\section{WHO Traditional Medicine (TRM) Strategy and Its} Evolution

Date: Saturday, October 30, 2004 (Concurrent Presentations) Presenter: Dr Seung Hoon Choi, Regional Adviser in Traditional Medicine, World Health Organization, Western Pacific Regional Office (Philippines).

\section{Acupuncture for Arthritis-Meta-analysis}

Date: Saturday, October 30, 2004 (Concurrent Presentations)

Presenter: Prof. Jin Zhigao, Professor Acupuncture Institute, China Academy of Traditional Chinese Medicine, Beijing, China.

Clinical and Experimental Research on Stroke Treated with 'Xing Nao Kai Qiao' Therapy

Date: Sunday, October 31, 2004 (Concurrent Presentation) Presenter: Prof. Shi Xuemin, Academician of Chinese Academy of Engineering; Honorary President of First Teaching Hospital of Tianjin College of Traditional Chinese Medicine, Tianjin, China.

\section{Strategy Future: The Need to Maintain a Strong Autonomous Professional Identity}

Date: Sunday, October 31, 2004 (Concurrent Presentation) Presenter: James Flowers, President, Australian Acupuncture and Chinese Medicine Association, Australia.

\section{Occupational Health and Safety for Chinese Medicine Practitioners}

Date: Sunday, October 31, 2004 (Concurrent Presentation) Presenter: Richard Keyuan Li, Vice-President WFAS, Australia.

\section{Research on the Key Question on Improving Acupuncture Clinical Assessment}

Date: Sunday, October 31, 2004 (Concurrent Presentation). Presenter: Prof. Liu Baoyan, WFAS Executive Member, Vice-President of China Academy of Traditional Chinese Medicine, China.

\section{Academic Awards}

The Academic Committee of the Conference selected one Best Overall Paper, one Best Research Paper, and one Best Paper on Clinical Application. Each winning author was awarded a Medpex Meridian Energy Analysis Device, model ME-Pro valued at USD\$9600 donated by Avazzia Inc (Plano, TX).

\section{Best Overall Paper}

Clinical and Experimental Research on Stroke Treated with 'Xing Nao Kai Qiao' Therapy

Xuemin Shi, Academician of the China Academy of Engineers and a Professor and honorary President of First Teaching 
Hospital of Tianjin University of Traditional Chinese Medicine, was the winning author of Best Overall Paper. Prof. Shi reported clinical observations and experimental results of Xing Nao Kai Qiao (activating the brain and opening the orifices) therapy on stroke, which had a very large sample size of more than 9000 stroke patients. The main acupuncture points used in the therapy were PC 6, DU 26 and SP 6. They found that the effective rate was $>98 \%$ in 9005 cases of stroke patients in different periods. The results demonstrated that the therapy can improve microcirculation, brain memory function and injury of reperfusion. They have also found that changes in exceedingly large micro-configuration, cranial transmission, free radicals, intracellular calcium ion, and DNA and RNA transcriptions are responsible for the acupuncture effects. They concluded that Xing Nao Kai Qiao is an effective acupuncture therapy for treatment of stroke patients.

\section{Best Research Paper}

Nitric Oxide Mediates Acupuncture-Induced

Cardiovascular Effects in the Dorsal Medulla-Thalamic

Tract

Sheng-Xing Ma, an Associate Professor from Harbor-UCLA Medical Center, David Geffen School of Medicine at the University of California Los Angeles (USA), received Best Research Paper award, for work which is an excellent example of the evidence-based research model for acupuncture practice. Dr Ma reported that L-arginine-derived nitric oxide (NO) in the brain pathway mediates cardiovascular and anti-hypertensive responses to electroacupuncture (EA) stimulation of ST 36 acupuncture point in rats. Results showed that EA ST 36 produced a decrease in mean arterial blood pressure (MAP) in rats. The MAP was significantly decreased by the second EA stimulation in established salt-induced hypertension but not significantly altered by short period EA. Dr Ma found that EA stimulation of point ST 36 induces expression of neuronal NO synthase in the gracile nucleus and nucleus tractus solitarius (NTS) regions. These results represent a novel discovery of how stimulation by acupuncture induces nNOS-NO in the gracile nucleus and NTS, which may modify central sympathetic functions and contribute to the hypotensive effects of the therapy.

\section{Best Paper on Clinical Application}

Plantar Fascitis-Another Approach

Stephen Janz, Chair of the National Academic Standards Committee for Traditional Chinese Medicine, discussed the enhanced clinical efficiency of treatment of Plantar Fascitis in a protocol extending beyond the conventional treatments using orthotics and steroid injections, which generally produce poor responses. His protocol increases the scope of corrective focus beyond the lower extremity, the local site of debilitating pathology, to a method which includes broader analysis and treatment of latent lower back pathology, as well as using additional extra acupuncture points around the heel of the foot.

\section{Significance of the Conference}

The WFAS 2004 Conference was valuable in many ways. First, it assembled a large international group of doctors and researchers with clinical emphasis on acupuncture and TCM as their standard practice. This promoted many stimulating discussions of methods, treatments and other related issues by a very broad group of leading acupuncture practitioners.

Second, WFAS 2004 was co-sponsored by the WHO, which actively supports well-defined strategic objectives globally, in order to strengthen traditional medical practice in both developing and developed countries. The WHOs Regional Strategy for Traditional Medicine in the Western Pacific (2001-2010) identifies major strategic objectives for traditional medicine, including formulation of national policies, establishment of uniform practice standards, promotion of evidence-based practice research, and policies to protect and conserve health resources.

Third, conference participants presented broad coverage of topics and methods, ranging from scholarly studies of traditional acupuncture-moxibustion practice through state-of-the-art physiologic studies utilizing powerful modern techniques such as cell biology, molecular biology, brain imaging and advanced statistical analyses. Establishing the scope and mechanisms of the effects of acupuncture practice on an evidence-based approach clearly can only benefit by bringing together the many practitioners who participated in WFAS 2004.

Received January 19, 2005; accepted February 9, 2005 


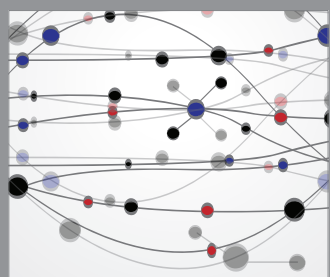

The Scientific World Journal
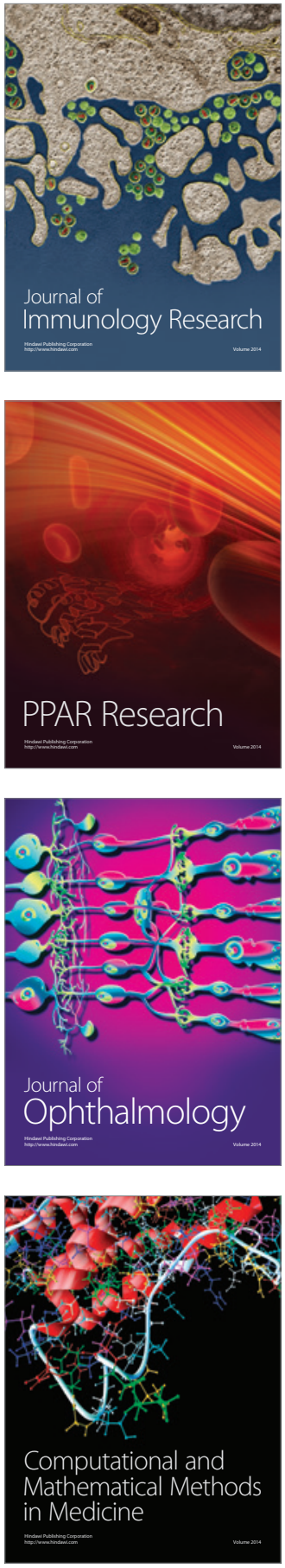

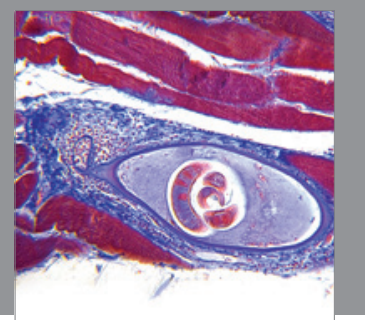

Gastroenterology

Research and Practice
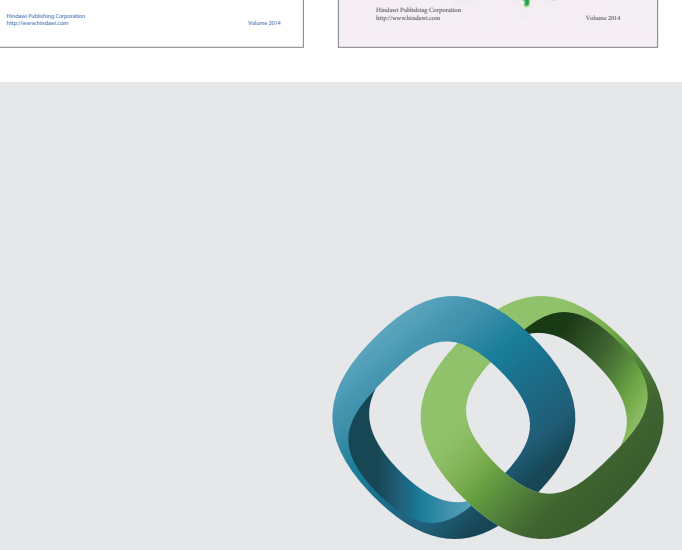

\section{Hindawi}

Submit your manuscripts at

http://www.hindawi.com
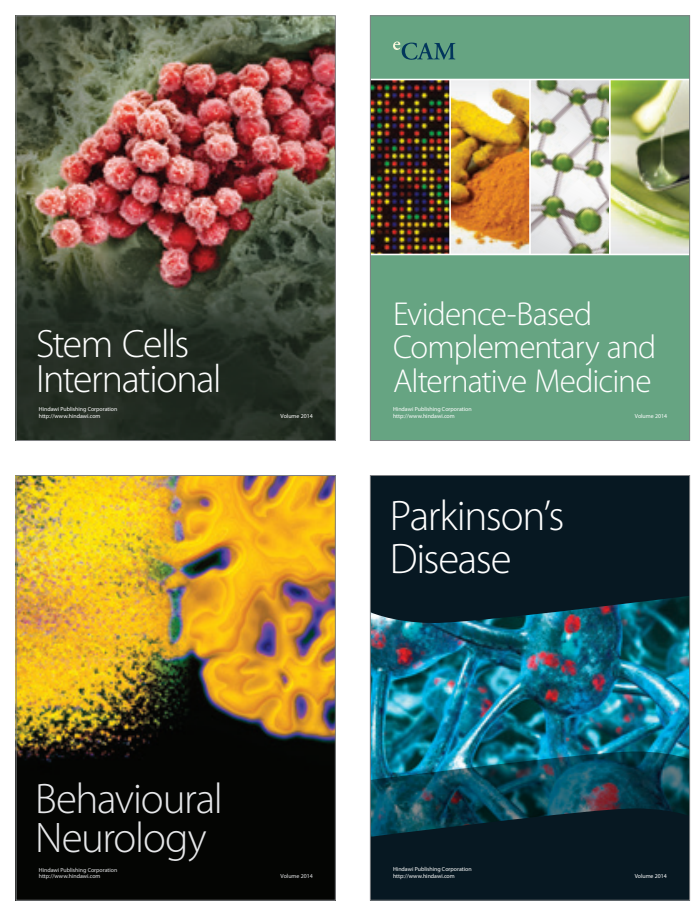

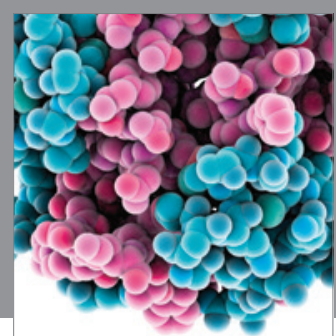

Journal of
Diabetes Research

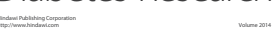

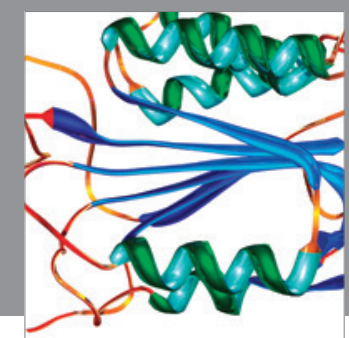

Disease Markers
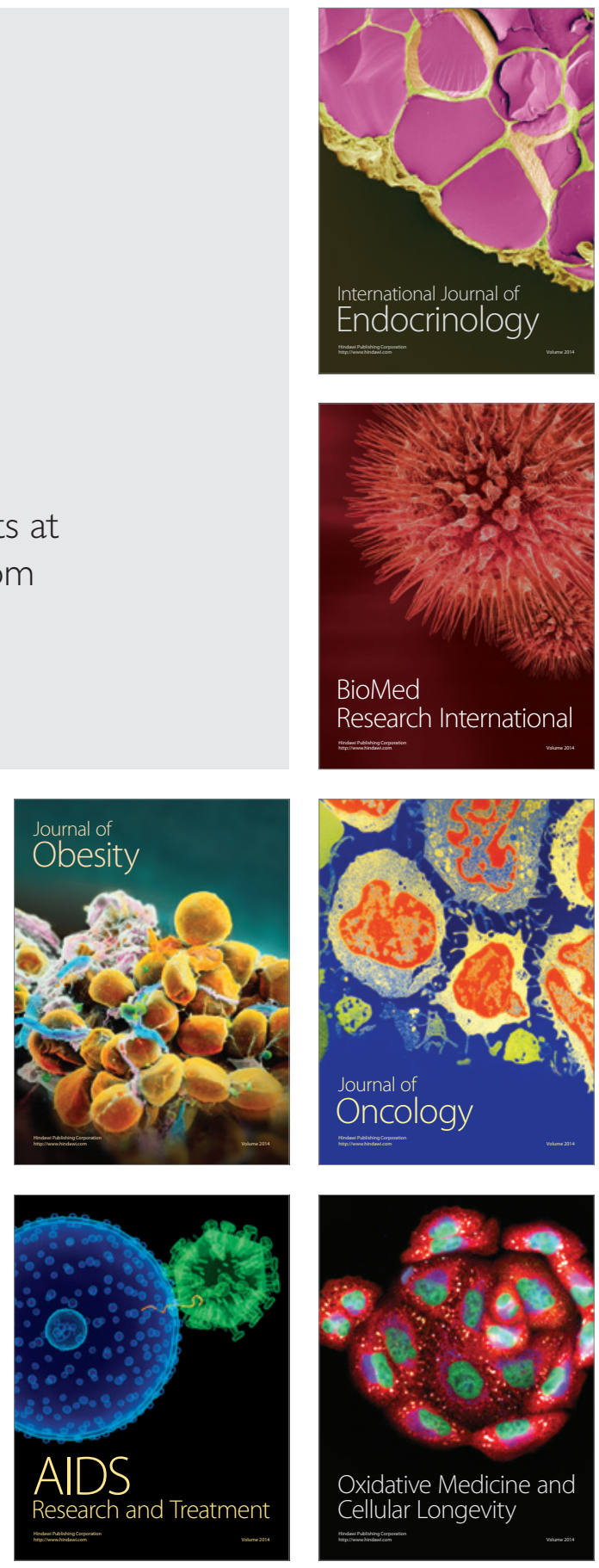